\title{
Integral transform solution of the Navier-Stokes equations in full cylindrical regions with streamfunction formulation ${ }^{\ddagger}$
}

\author{
C. A. M. Silva ${ }^{1}$, E. N. Macêdo ${ }^{1}$, J. N. N. Quaresma ${ }^{1}$, L. M. Pereira ${ }^{2}$ \\ and R. M. $\operatorname{Cotta}^{3, *, \dagger}$ \\ ${ }^{1}$ School of Chemical Engineering, FEQ, Universidade Federal do Pará, UFPA, Campus Universitário do Guamá, \\ 66075-110 Belém, PA, Brazil \\ ${ }^{2}$ Mechanical Engineering Department, CEMEC, Universidade Federal do Vale do São Francisco, UNIVASF, \\ Campus Universitário de Petrolina, 506304-205 Petrolina, PE, Brazil \\ ${ }^{3}$ Mechanical Engineering Department-POLI/COPPE, Universidade Federal do Rio de Janeiro, UFRJ, \\ Cx. Postal 68503-Cidade Universitária, 21945-970 Rio de Janeiro, RJ, Brazil
}

\begin{abstract}
SUMMARY
A hybrid numerical-analytical solution based on the generalized integral transform technique is proposed to handle the two-dimensional Navier-Stokes equations in cylindrical coordinates, expressed in terms of the streamfunction-only formulation. The proposed methodology is illustrated in solving steady-state incompressible laminar flow of Newtonian fluids in the developing region of a circular tube. The flow modeling also considers two limiting inlet conditions, namely, uniform velocity profile representing a parallel flow, and zero vorticity that characterizes irrotational inlet flow. The integral transform analysis for such a full cylindrical region brings up singularities at the channel centerline, and, as previously described in a work dealing with the boundary-layer formulation, a way to alleviate this difficulty is to adopt a recently introduced fourth-order eigenvalue problem as the basis for the eigenfunction expansion. A thorough convergence analysis of the proposed expansion is then undertaken, for different values of Reynolds number, and a set of reference results for the velocity distributions and friction factors are then presented in tabular and graphical forms. Copyright (C) 2009 John Wiley \& Sons, Ltd.
\end{abstract}

Received 25 July 2008; Revised 21 October 2008; Accepted 12 December 2008

KEY WORDS: Navier-Stokes equations; hydrodynamically developing flow; circular tubes; integral transforms; hybrid methods; friction factor

\section{INTRODUCTION}

The solution of the Navier-Stokes equations remains a major research topic in computational mechanics in light of the marked nonlinear characteristics associated with them. Applications involving flows governed by such formulation have been used to test the consistency and robustness of different numerical solution methodologies. Alternative tools based on spectral-type hybrid numerical-analytical methodologies, contrasting with the more straightforward treatment of the

\footnotetext{
${ }^{*}$ Correspondence to: R. M. Cotta, Mechanical Engineering Department-POLI/COPPE, Universidade Federal do Rio de Janeiro, UFRJ, Cx. Postal 68503-Cidade Universitária, 21945-970 Rio de Janeiro, RJ, Brazil.

${ }^{\dagger}$ E-mail: cotta@mecanica.coppe.ufrj.br, cottarenato@terra.com.br

${ }^{\ddagger}$ This article was published online on 28 January 2009. An error was subsequently identified. This notice is included in the online and print versions to indicate that both have been corrected [2 March 2009].
}

Contract/grant sponsor: $\mathrm{CNPq}$

Contract/grant sponsor: CENPES-Petrobras

Copyright (C) 2009 John Wiley \& Sons, Ltd. 
conventional numerical approaches for such partial differential models, were also developed and are especially suitable for benchmark and covalidation purposes [1].

Along the years, the literature reveals the progressive development of hybrid schemes based on eigenfunction expansions, which recently, due to the development of symbolic computation platforms, have became more attractive due to the reduction on the analytical development effort. Among such hybrid techniques, one may recall the generalized integral transform technique (GITT) [2-8] that was successfully extended and became applicable to a large number of problems in transport phenomena, including fluid flow problems under either the boundary layer or the full Navier-Stokes formulations [9-28]. In such contributions, the preference for the streamfunctiononly formulation in two-dimensional situations is notorious, in light of the elimination of the pressure field and automatic satisfaction of the continuity equation. The application of GITT to the streamfunction-only formulation of two-dimensional flow problems leads to an eigenfunction expansion resulting from the analytical solution of an appropriate fourth-order eigenvalue problem related to the linear biharmonic equation. Problems related to the Cartesian coordinates system were more frequently studied in comparison to those propositions of eigenfunction expansions in the cylindrical coordinates system $[20,25,28]$, possibly due to the inherent difficulties in avoiding the singularities of the fourth-order eigenvalue problem at the circular duct centerline for a full cylindrical region. These difficulties were circumvented in a recent work dealing with the boundarylayer equations [28], by adopting an also recently introduced eigenvalue problem [29] which accounts for the singularities at the central radial position.

The flow in the entrance region of a circular tube is still considered an important test problem for assessment of different methodologies focused on the solution of internal fluid flow and convective heat transfer [30,31]. A literature survey reveals classical works that dealt with the solution of the Navier-Stokes equations in an approximate analytical way or through purely numerical techniques, such as the linearization approach by Sparrow et al. [32] and the early solutions with finite differences by Hornbeck [33] and Liu [34]. In addition, Friedmann et al. [35] investigated hydrodynamically developing flow in a circular tube at low and moderate Reynolds numbers, also using a finite difference scheme to numerically solve the problem. They obtained numerical velocity profiles and an analytic solution for the limiting case of vanishing Reynolds number, performing several comparisons with results then available in the literature.

In this context, the GITT approach with its intrinsic characteristic of finding solutions with automatic global error control, opened up an alternative perspective in benchmarking and covalidation for such classical test problems [2-8]. At the present stage, the GITT methodology was already successfully employed in the solution of the boundary-layer formulation version of this same problem, by adopting an appropriate fourth-order eigenvalue problem in the cylindrical coordinates system that could exactly deal with the singularities of the Bessel functions at the tube centerline $[28,29]$. The resulting system of transformed streamfunctions then offered an ordinary differential equation (ODE) system as an initial value problem along the axial coordinate, due to the parabolic nature of the modeling equations. Following the same line of research, the present work is thus aimed at utilizing the ideas in the GITT methodology to construct a hybrid analytical-numerical solution, now for the full Navier-Stokes equations. By adopting the same fourth-order eigenvalue problem, the transformed streamfunctions system results in a fourth-order boundary value problem to be numerically solved along the axial coordinate. Computation of the velocity field and friction factor along the entrance region is performed for various values of Reynolds number, following a thorough convergence analysis of the eigenfunction expansions. Critical comparisons with earlier published works are also performed to allow for a covalidation against the present GITT results.

\section{ANALYSIS}

Laminar flow of a Newtonian fluid is considered along the developing region of a circular tube as shown in Figure 1. The flow is assumed to be incompressible and physical properties are considered constant. The steady two-dimensional continuity equation, together with the full Navier-Stokes 


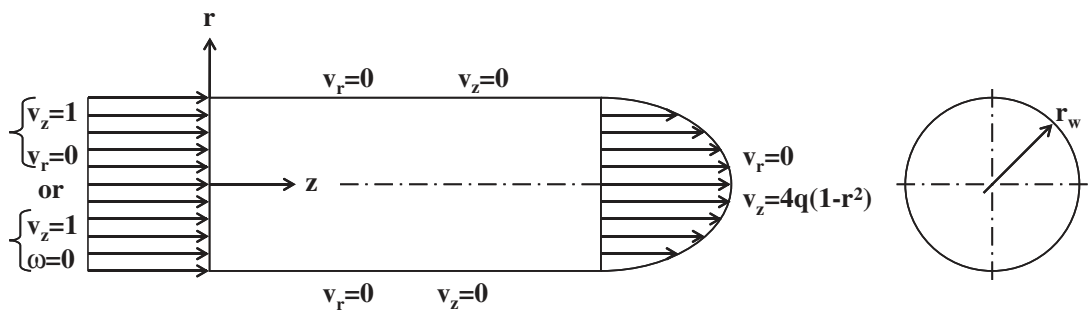

Figure 1. Geometry and coordinates system for hydrodynamically developing flow in circular duct.

equations in cylindrical coordinates, are used to model the flow. Two different inlet conditions are analyzed for a known longitudinal velocity component $v_{z}$ at the channel entrance, i.e. parallel inlet flow $\left(v_{r}=0\right)$ and irrotational inlet flow $\left(\partial v_{r} / \partial z=0\right)$. Fully developed flow conditions are attained at a sufficiently large channel length, recovering the parabolic flow structure for the velocity field. Therefore, the modeling of this flow field, in the region $0<r<1$ and $z>0$, in terms of the streamfunction-only formulation such as described in [28] is written, in dimensionless form, as:

$$
\begin{gathered}
\frac{1}{r} \frac{\partial \phi}{\partial z}\left[\frac{\partial}{\partial r}\left(E^{2} \phi\right)-\frac{2}{r}\left(E^{2} \phi\right)\right]-\frac{1}{r} \frac{\mathrm{d} \psi \infty}{\mathrm{d} r} \frac{\partial}{\partial z}\left(E^{2} \phi\right)-\frac{1}{r} \frac{\partial \phi}{\partial r} \frac{\partial}{\partial z}\left(E^{2} \phi\right)=\frac{2}{R e}\left(E^{4} \phi\right) \\
\lim _{r \rightarrow 0}\left[\frac{\phi(r, z)}{r}\right]=0 \\
\lim _{r \rightarrow 0}\left\{\frac{\partial}{\partial r}\left[\frac{1}{r} \frac{\partial \phi(r, z)}{\partial r}\right]\right\}=0 \\
\frac{\phi(1, z)=0}{\frac{\partial \phi(1, z)}{\partial r}=0} \\
\frac{\phi(r, 0)}{r}=\frac{r}{2}\left(1-r^{2}\right) \\
\frac{\partial(r, 0)}{\partial z}=0 \quad \text { for parallel flow or } \\
\frac{\partial^{2} \phi(r, 0)}{\partial z^{2}}=0 \quad \text { for irrotational flow } \\
\frac{\phi(r, \infty)}{r}=0 \\
\frac{\partial \phi(r, \infty)}{\partial z}=0
\end{gathered}
$$

where the operators $E^{2}$ and $E^{4}$ are defined as

$$
\begin{aligned}
& E^{2}=\frac{\partial^{2}}{\partial r^{2}}-\frac{1}{r} \frac{\partial}{\partial r}+\frac{\partial^{2}}{\partial z^{2}} \\
& E^{4}=\frac{\partial^{4}}{\partial r^{4}}-\frac{2}{r} \frac{\partial^{3}}{\partial r^{3}}+\frac{3}{r^{2}} \frac{\partial^{2}}{\partial r^{2}}-\frac{3}{r^{3}} \frac{\partial}{\partial r}-\frac{2}{r} \frac{\partial^{3}}{\partial r \partial z^{2}}+2 \frac{\partial^{4}}{\partial r^{2} \partial z^{2}}+\frac{\partial^{4}}{\partial z^{4}}
\end{aligned}
$$

The above streamfunction-only formulation, Equations (1)-(9), has been preferred in the integral transform solution of flow problems in light of the elimination of the pressure field and for automatically satisfying the continuity equation. Also, the definition of the functions $\psi_{\infty}(r) \equiv \psi(\infty, r)$ 
and $\phi(r, z)$, which represent, respectively, the streamfunction at the fully developed region and the filtered potential to be actually solved for, makes it possible to homogenize the problem boundary conditions in the radial coordinate. Such functions are related to the original streamfunction in the following form:

$$
\begin{aligned}
& \psi(r, z)=\psi_{\infty}(r)+\phi(r, z) \\
& \psi_{\infty}(r)=r^{2}\left(\frac{r^{2}}{2}-1\right)
\end{aligned}
$$

The velocity components in the longitudinal $(z)$ and radial $(r)$ directions are given in terms of the streamfunction, respectively, as

$$
\begin{aligned}
& v_{r}=\frac{1}{r} \frac{\partial \psi}{\partial z}=\frac{1}{r} \frac{\partial \phi}{\partial z} \\
& v_{z}=-\frac{1}{r} \frac{\partial \psi}{\partial r}=-\frac{1}{r}\left(\frac{\mathrm{d} \psi_{\infty}}{\mathrm{d} r}+\frac{\partial \phi}{\partial r}\right)
\end{aligned}
$$

The dimensionless groups employed in the above equations are defined as:

$$
r=r^{*} / r_{\mathrm{w}}, \quad z=z^{*} / r_{\mathrm{w}}, \quad v_{r}=v_{r}^{*} / u_{0}, \quad v_{z}=v_{z}^{*} / u_{0}, \quad p=p^{*} / \rho u_{0}^{2}, \quad R e=2 u_{0} r_{\mathrm{w}} / v
$$

In order to obtain the solution of the PDE system given by Equations (1)-(9) through the GITT approach, the following integral transform pair for the streamfunction field is developed [28]:

$$
\begin{aligned}
\bar{\phi}_{i}(z) & =-\frac{1}{N_{i}} \int_{0}^{1}\left(E X_{i}(r)\right) \phi(r, z) \frac{\mathrm{d} r}{r} \quad \text { transform } \\
\phi(r, z) & =\sum_{i=1}^{\infty} X_{i}(r) \bar{\phi}_{i}(z) \quad \text { inverse }
\end{aligned}
$$

where $X_{i}(r)$ are the eigenfunctions of an appropriate eigenvalue problem as discussed in details in References [28,29], which is given as

$$
\begin{aligned}
E^{4} X_{i}(r) & =-\lambda_{i}^{2} E^{2} X_{i}(r) \\
\lim _{r \rightarrow 0}\left[\frac{X_{i}(r)}{r}\right] & =0 \\
\lim _{r \rightarrow 0}\left\{\frac{\mathrm{d}}{\mathrm{d} r}\left[\frac{1}{r} \frac{\mathrm{d} X_{i}(r)}{\mathrm{d} r}\right]\right\} & =0 \\
X_{i}(1) & =0 \\
\frac{\mathrm{d} X_{i}(1)}{\mathrm{d} r} & =0
\end{aligned}
$$

where,

$$
\begin{aligned}
& E^{2}=r \frac{\mathrm{d}}{\mathrm{d} r}\left(\frac{1}{r} \frac{\mathrm{d}}{\mathrm{d} r}\right)=\frac{\mathrm{d}^{2}}{\mathrm{~d} r^{2}}-\frac{1}{r} \frac{\mathrm{d}}{\mathrm{d} r} \\
& E^{4}=E^{2}\left(E^{2}\right)=\frac{\mathrm{d}^{4}}{\mathrm{~d} r^{4}}-\frac{2}{r} \frac{\mathrm{d}^{3}}{\mathrm{~d} r^{3}}+\frac{3}{r^{2}} \frac{\mathrm{d}^{2}}{\mathrm{~d} r^{2}}-\frac{3}{r^{3}} \frac{\mathrm{d}}{\mathrm{d} r}
\end{aligned}
$$


The operators $E^{2}$ and $E^{4}$ above are just the reduced forms of the previously defined ones in Equations (10), (11), as applied to a function of one single argument, $r$, and whose solution is of the following form:

$$
X_{i}(r)=r^{2}-\frac{r J_{1}\left(\lambda_{i} r\right)}{J_{1}\left(\lambda_{i}\right)}
$$

The eigenvalues $\lambda_{i}$ 's are computed from the following transcendental equation:

$$
J_{2}\left(\lambda_{i}\right)=0, \quad i=1,2,3, \ldots
$$

The eigenfunctions satisfy the following orthogonality property:

$$
\int_{0}^{1} \frac{\mathrm{d} X_{i}(r)}{\mathrm{d} r} \frac{\mathrm{d} X_{j}(r)}{\mathrm{d} r} \frac{\mathrm{d} r}{r}=-\int_{0}^{1} X_{i}(r)\left[E^{2} X_{j}(r)\right] \frac{\mathrm{d} r}{r}= \begin{cases}0, & i \neq j \\ N_{i}, & i=j\end{cases}
$$

The normalization integral $N_{i}$ is then computed from:

$$
N_{i}=\int_{0}^{1} \frac{1}{r}\left[\frac{\mathrm{d} X_{i}(r)}{\mathrm{d} r}\right]^{2} \mathrm{~d} r=\frac{\lambda_{i}^{2}}{2}
$$

Now, the next step is thus to accomplish the integral transformation of the original partial differential system given by Equations (1)-(9). For this purpose, Equation (1), followed by the inlet an outflow boundary conditions given by Equations (6)-(9), are multiplied by $\left[X_{i}(r) / r\right]$, integrated over the domain in $r[0,1]$, and the inverse formula given by Equation (18) is employed. After the usual manipulations, the following coupled ordinary differential system results, for the calculation of the transformed potentials $\bar{\phi}_{i}(z)$ :

$$
\begin{aligned}
\sum_{j=1}^{\infty} A_{i j} & \frac{\mathrm{d}^{4} \bar{\phi}_{j}(z)}{\mathrm{d} z^{4}}-2 \frac{\mathrm{d}^{2} \bar{\phi}_{i}(z)}{\mathrm{d} z^{2}} N_{i}+\lambda_{i}^{2} \bar{\phi}_{i}(z) N_{i} \\
= & \frac{R e}{2}\left[\sum_{k=1}^{\infty} \sum_{j=1}^{\infty}\left(B_{i j k} \bar{\phi}_{j}(z) \frac{\mathrm{d} \bar{\phi}_{k}(z)}{\mathrm{d} z}+C_{i j k} \frac{\mathrm{d} \bar{\phi}_{j}(z)}{\mathrm{d} z} \frac{\mathrm{d}^{2} \bar{\phi}_{k}(z)}{\mathrm{d} z^{2}}+D_{i j k} \bar{\phi}_{j}(z) \frac{\mathrm{d}^{3} \bar{\phi}_{k}(z)}{\mathrm{d} z^{3}}\right)\right. \\
& \left.+\sum_{j=1}^{\infty}\left(E_{i j} \frac{\mathrm{d} \bar{\phi}_{j}(z)}{\mathrm{d} z}+F_{i j} \frac{\mathrm{d}^{3} \bar{\phi}_{j}(z)}{\mathrm{d} z^{3}}\right)\right], \quad i=1,2,3, \ldots
\end{aligned}
$$

with boundary conditions

$$
\begin{aligned}
\bar{\phi}_{i}(0) & =\bar{g}_{i} \\
\frac{\mathrm{d} \bar{\phi}_{i}(0)}{\mathrm{d} z} & =0 \quad \text { for parallel flow or } \\
\frac{\mathrm{d}^{2} \bar{\phi}_{i}(0)}{\mathrm{d} z^{2}} & =0 \quad \text { for irrotational flow } \\
\bar{\phi}_{i}(\infty) & =0 \\
\frac{\mathrm{d} \bar{\phi}_{i}(\infty)}{\mathrm{d} z} & =0
\end{aligned}
$$


where the coefficients of the ordinary differential system are given by the following expressions:

$$
\begin{aligned}
A_{i j}= & \int_{0}^{1} X_{i}(r) X_{j}(r) \frac{\mathrm{d} r}{r} \\
B_{i j k}= & \int_{0}^{1} X_{i}(r)\left[\frac{X_{j}^{\prime \prime \prime}(r) X_{k}(r)}{r^{2}}-3 \frac{X_{j}^{\prime \prime}(r) X_{k}(r)}{r^{3}}+3 \frac{X_{j}^{\prime}(r) X_{k}(r)}{r^{4}}\right. \\
& \left.-\frac{X_{j}^{\prime}(r) X_{k}^{\prime \prime}(r)}{r^{2}}+\frac{\left.X_{j}^{\prime}(r) X_{k}^{\prime}(r)\right]}{r^{3}}\right] \mathrm{d} r \\
C_{i j k}= & \int_{0}^{1} X_{i}(r)\left[\frac{X_{j}(r) X_{k}^{\prime}(r)}{r^{2}}-2 \frac{X_{j}(r) X_{k}(r)}{r^{3}}\right] \mathrm{d} r \\
D_{i j k}= & -\int_{0}^{1} \frac{X_{i}(r) X_{j}^{\prime}(r) X_{k}(r)}{r^{2}} \mathrm{~d} r \\
E_{i j}= & \int_{0}^{1} X_{i}(r)\left[\frac{X_{j}^{\prime}(r)}{r^{3}}-\frac{X_{j}^{\prime \prime}(r)}{r^{2}}\right] \frac{\mathrm{d} \psi_{\infty}(r)}{\mathrm{d} r} \mathrm{~d} r \\
F_{i j}= & -\int_{0}^{1} \frac{X_{i}(r) X_{j}(r)}{r^{2}} \frac{\mathrm{d} \psi_{\infty}(r)}{\mathrm{d} r} \mathrm{~d} r \\
\bar{g}_{i}= & \frac{1}{N_{i}} \int_{0}^{1} \frac{r^{2}}{2}\left(r^{2}-1\right)\left(E X_{j}(r)\right) \frac{\mathrm{d} r}{r}
\end{aligned}
$$

In order to numerically handle the ODE system given by Equations (30)-(34), we have here employed subroutine DBVPFD of the IMSL Library [36]. It is then necessary to truncate the infinite series in a sufficiently large number of terms (NV) so as to guarantee the requested overall relative error in obtaining the original potentials. This subroutine solves a (parameterized) system of differential equations with boundary conditions at two points, using a variable order, variable step size finite difference method with deferred corrections. It also provides the important feature of automatically controlling the relative error in the solution of the ODEs system, allowing the user to establish error targets for the transformed potentials.

Once the transformed potentials $\bar{\phi}_{i}(z)$ are available, the velocity field is obtained from the definition of the streamfunction given by Equations (14) and (15), after introducing the inverse formula (18). In addition, the product of the Fanning friction factor-Reynolds number, $\mathrm{f} R e$, is obtained from its usual definition, and the following working expressions are constructed:

$$
\begin{aligned}
v_{r}(r, z) & =\sum_{i=1}^{\infty} \frac{X_{i}(r)}{r} \frac{\mathrm{d} \bar{\phi}_{i}(z)}{\mathrm{d} z} \\
v_{z}(r, z) & =2\left(1-r^{2}\right)-\sum_{i=1}^{\infty} \frac{X_{i}^{\prime}(r)}{r} \bar{\phi}_{i}(z) \\
\mathrm{f} R e & =16+4 \sum_{i=1}^{\infty} \frac{\mathrm{d}}{\mathrm{d} r}\left[\frac{1}{r} \frac{\mathrm{d} X_{i}(1)}{\mathrm{d} r}\right] \bar{\phi}_{i}(z)
\end{aligned}
$$

To make use of subroutine DBVPFD, it is necessary to rewrite the fourth-order system as a first order one, through the following transformations in terms of a solution vector:

$$
\begin{aligned}
\bar{\phi}_{i} & =W_{i} \\
\frac{\mathrm{d} \bar{\phi}_{i}}{\mathrm{~d} z} & =\frac{\mathrm{d} W_{i}}{\mathrm{~d} z}=W_{N+i}
\end{aligned}
$$




$$
\begin{aligned}
& \frac{\mathrm{d}^{2} \bar{\phi}_{i}}{\mathrm{~d} z^{2}}=\frac{\mathrm{d}}{\mathrm{d} z}\left(\frac{\mathrm{d} \bar{\phi}_{i}}{\mathrm{~d} z}\right)=\frac{\mathrm{d} W_{N+i}}{\mathrm{~d} z}=W_{2 N+i} \\
& \frac{\mathrm{d}^{3} \bar{\phi}_{i}}{\mathrm{~d} z^{3}}=\frac{\mathrm{d}}{\mathrm{d} z}\left(\frac{\mathrm{d}^{2} \bar{\phi}_{i}}{\mathrm{~d} z^{2}}\right)=\frac{\mathrm{d} W_{2 N+i}}{\mathrm{~d} z}=W_{3 N+i} \\
& \frac{\mathrm{d}^{4} \bar{\phi}_{i}}{\mathrm{~d} z^{4}}=\frac{\mathrm{d}}{\mathrm{d} z}\left(\frac{\mathrm{d}^{3} \bar{\phi}_{i}}{\mathrm{~d} z^{3}}\right)=\frac{\mathrm{d} W_{3 N+i}}{\mathrm{~d} z}
\end{aligned}
$$

Therefore, introducing the definitions above in Equations (30)-(34) and truncating the infinite series in a sufficiently large truncation order, NV, the transformed system takes the following form:

$$
\begin{gathered}
\sum_{j=1}^{\mathrm{NV}} A_{i j} \frac{\mathrm{d} W_{3 \mathrm{NV}+j} \mathrm{~d} z \quad}{=}-\lambda_{i}^{2} W_{i} N_{i}+2 W_{2 \mathrm{NV}+i} N_{i} \\
+\frac{R e}{2}\left[\sum _ { k = 1 } ^ { \mathrm { NV } } \sum _ { j = 1 } ^ { \mathrm { NV } } \left(B_{i j k} W_{j} W_{\mathrm{NV}+k}+C_{i j k} W_{\mathrm{NV}+j} W_{2 \mathrm{NV}+k}\right.\right. \\
\left.\left.+D_{i j k} W_{j} W_{3 \mathrm{NV}+k}\right)+\sum_{j=1}^{\mathrm{NV}}\left(E_{i j} W_{\mathrm{NV}+j}+F_{i j} W_{3 \mathrm{NV}+j}\right)\right] \\
W_{i}(0)=\bar{g}_{i} \\
W_{\mathrm{NV}+i}(0)=0 \quad \text { for parallel flow or } \\
W_{2 \mathrm{NV}+i}(0)=0 \quad \text { for irrotational flow } \\
W_{i}(\infty)=0 \\
W_{\mathrm{NV}+i}(\infty)=0
\end{gathered}
$$

The above coupled ordinary differential system for the transformed potentials $\bar{\phi}_{i}(z)$ has boundary conditions specified at infinity, and it is also convenient to propose a domain transformation to map the longitudinal coordinate in the following form: $z \in[0, \infty) \rightarrow \eta \in[0,1]$. This can be achieved for instance by the following choice:

$$
\begin{aligned}
\eta & =1-\mathrm{e}^{-c z} \\
\frac{\mathrm{d} \eta}{\mathrm{d} z} & =c(1-\eta) \\
\frac{\mathrm{d}()}{\mathrm{d} z} & =\frac{\mathrm{d} \eta}{\mathrm{d} z} \frac{\mathrm{d}()}{\mathrm{d} \eta}
\end{aligned}
$$

Therefore, the system to be numerically handled by subroutine DBVPFD is written as:

$$
\begin{array}{r}
\frac{\mathrm{d} W_{i}}{\mathrm{~d} \eta}=\frac{W_{\mathrm{NV}+i}}{\left(\frac{\mathrm{d} \eta}{\mathrm{d} z}\right)} \\
\frac{\mathrm{d} W_{\mathrm{NV}+i}}{\mathrm{~d} \eta}=\frac{W_{2 \mathrm{NV}+i}}{\left(\frac{\mathrm{d} \eta}{\mathrm{d} z}\right)} \\
\frac{\mathrm{d} W_{2 \mathrm{NV}+i}}{\mathrm{~d} \eta}=\frac{W_{3 \mathrm{NV}+i}}{\left(\frac{\mathrm{d} \eta}{\mathrm{d} z}\right)}
\end{array}
$$




$$
\begin{aligned}
\sum_{j=1}^{\mathrm{NV}} A_{i j} \frac{\mathrm{d} W_{3 \mathrm{NV}+j}}{\mathrm{~d} \eta}= & \frac{1}{\left(\frac{\mathrm{d} \eta}{\mathrm{d} z}\right)}\left\{-\lambda_{i}^{2} W_{i} N_{i}+2 W_{2 \mathrm{NV}+i} N_{i}+\frac{R e}{2}\left[\sum _ { k = 1 } ^ { \mathrm { NV } } \sum _ { j = 1 } ^ { \mathrm { NV } } \left(B_{i j k} W_{j} W_{\mathrm{NV}+k}\right.\right.\right. \\
& \left.+C_{i j k} W_{\mathrm{NV}+j} W_{2 \mathrm{NV}+k}+D_{i j k} W_{j} W_{3 \mathrm{NV}+k}\right) \\
& \left.\left.+\sum_{j=1}^{\mathrm{NV}}\left(E_{i j} W_{\mathrm{NV}+j}+F_{i j} W_{3 \mathrm{NV}+j}\right)\right]\right\}
\end{aligned}
$$

$$
W_{i}(0)=\bar{g}_{i}
$$

$$
\begin{aligned}
W_{\mathrm{NV}+i}(0)=0 & \text { for parallel flow or } \\
W_{2 \mathrm{NV}+i}(0)=0 & \text { for irrotational flow } \\
W_{i}(1)=0 & \\
W_{\mathrm{NV}+i}(1)=0 &
\end{aligned}
$$

The Jacobian matrix required by the numerical algorithm is constructed with the derivatives of the transformed system with relation to each one of the components of the solution vector $\mathrm{W}$, as:

$$
\left.\left.\begin{array}{rl}
\frac{\partial\left(\frac{\mathrm{d} W_{i}}{\mathrm{~d} \eta}\right)}{\partial W_{\mathrm{NV}+i}}=\frac{1}{\left(\frac{\mathrm{d} \eta}{\mathrm{d} z}\right)} & \frac{\partial\left(\frac{\left.\mathrm{d} W_{\mathrm{NV}+i}\right)}{\mathrm{d} \eta}\right)}{\partial W_{2 \mathrm{NV}+i}}=\frac{1}{\left(\frac{\mathrm{d} \eta}{\mathrm{d} z}\right)} \\
\frac{\partial\left(\frac{\mathrm{d} W_{2 \mathrm{NV}+i}}{\mathrm{~d} \eta}\right)}{\partial W_{3 \mathrm{NV}+i}} & =\frac{1}{\left(\frac{\mathrm{d} \eta}{\mathrm{d} z}\right)} \\
\sum_{j=1}^{\mathrm{NV}} A_{i j} \frac{\partial\left(\frac{\mathrm{d} W_{3 \mathrm{NV}+j}}{\mathrm{~d} \eta}\right)}{\partial W_{m}} & =\frac{1}{\left(\frac{\mathrm{d} \eta}{\mathrm{d} z}\right)}\left\{-\lambda_{i}^{2} \delta_{i m} N_{i}+\frac{R e}{2}\left[\sum_{k=1}^{\mathrm{NV}}\left(B_{i m k} W_{\mathrm{NV}+k}+D_{i m k} W_{3 \mathrm{NV}+k}\right)\right]\right\} \\
\sum_{j=1}^{\mathrm{NV}} A_{i j} \frac{\partial\left(\frac{\mathrm{d} W_{3 \mathrm{NV}+j}}{\mathrm{~d} \eta}\right)}{\partial W_{\mathrm{NV}+m}} & =\frac{1}{\left(\frac{\mathrm{d} \eta}{\mathrm{d} z}\right)}\left\{\frac{\operatorname{Re}}{2}\left[\sum_{j=1}^{\mathrm{NV}} B_{i j m} W_{j}+\sum_{k=1}^{\mathrm{NV}} C_{i m k} W_{2 \mathrm{NV}+k}+E_{i m}\right]\right\} \\
\sum_{j=1} A_{i j} \frac{\partial\left(\frac{\mathrm{d} W_{3 \mathrm{NV}+j}}{\mathrm{~d} \eta}\right)}{\partial W_{2 \mathrm{NV}+m}} & =\frac{1}{\left(\frac{\mathrm{d} \eta}{\mathrm{d} z}\right)}\left\{2 \delta_{i m} N_{i}+\frac{\operatorname{Re}}{2}\left[\sum_{j=1}^{\mathrm{NV}}\left(C_{i j m} W_{\mathrm{NV}+j}\right)\right]\right\}
\end{array}\right]\right\}
$$




$$
\begin{aligned}
\sum_{j=1}^{\mathrm{NV}} A_{i j} \frac{\partial\left(\frac{\mathrm{d} W_{3 \mathrm{NV}+j}}{\mathrm{~d} \eta}\right)}{\partial W_{3 \mathrm{NV}+m}}= & \frac{1}{\left(\frac{\mathrm{d} \eta}{\mathrm{d} z}\right)}\left\{\frac{\operatorname{Re}}{2}\left[\sum_{j=1}^{\mathrm{NV}} D_{i j m} W_{j}+F_{i m}\right]\right\} \\
& i=1,2,3, \ldots, \mathrm{NV} ; m=1,2,3, \ldots, \mathrm{NV}
\end{aligned}
$$

\section{RESULTS AND DISCUSSION}

Numerical results for the velocity profiles and the product of the Fanning friction factor-Reynolds number were produced along the entrance region of a circular tube, for two types of boundary

Table I. Convergence behavior of the longitudinal velocity component along the centerline of the duct for

\begin{tabular}{|c|c|c|c|c|c|c|c|c|c|c|}
\hline \multirow[b]{2}{*}{ NV } & \multicolumn{10}{|c|}{$Z$} \\
\hline & 0.05 & 0.1 & 0.15 & 0.2 & 0.25 & 0.4 & 0.6 & 0.8 & 1.0 & 1.7 \\
\hline \multicolumn{11}{|l|}{$R e=0 ; v_{z}(0, z)$} \\
\hline 4 & 1.349 & 1.301 & 1.282 & 1.296 & 1.333 & 1.509 & 1.733 & 1.875 & 1.948 & 1.999 \\
\hline 8 & 1.207 & 1.159 & 1.167 & 1.211 & 1.272 & 1.483 & 1.723 & 1.871 & 1.947 & 1.999 \\
\hline 12 & 1.136 & 1.105 & 1.134 & 1.190 & 1.257 & 1.475 & 1.720 & 1.870 & 1.946 & 1.999 \\
\hline 16 & 1.095 & 1.082 & 1.122 & 1.181 & 1.250 & 1.471 & 1.717 & 1.869 & 1.946 & 1.999 \\
\hline 20 & 1.069 & 1.071 & 1.116 & 1.177 & 1.246 & 1.469 & 1.716 & 1.868 & 1.946 & 1.999 \\
\hline 24 & 1.053 & 1.065 & 1.112 & 1.174 & 1.244 & 1.467 & 1.715 & 1.868 & 1.945 & 1.999 \\
\hline 28 & 1.043 & 1.062 & 1.110 & 1.172 & 1.242 & 1.466 & 1.715 & 1.868 & 1.945 & 1.999 \\
\hline 32 & 1.036 & 1.059 & 1.108 & 1.170 & 1.240 & 1.465 & 1.714 & 1.867 & 1.945 & 1.999 \\
\hline 36 & 1.031 & 1.057 & 1.107 & 1.169 & 1.239 & 1.464 & 1.714 & 1.867 & 1.945 & 1.999 \\
\hline 40 & 1.028 & 1.056 & 1.106 & 1.168 & 1.238 & 1.463 & 1.713 & 1.867 & 1.945 & 1.999 \\
\hline 44 & 1.025 & 1.055 & 1.105 & 1.167 & 1.238 & 1.463 & 1.713 & 1.867 & 1.945 & 1.999 \\
\hline 48 & 1.024 & 1.054 & 1.104 & 1.167 & 1.237 & 1.462 & 1.713 & 1.867 & 1.945 & 1.999 \\
\hline \multirow[t]{2}{*}{ Reference [35] } & 1.0122 & 1.0458 & 1.0963 & 1.1593 & 1.2304 & 1.46 & 1.71 & 1.87 & 1.91 & 2.00 \\
\hline & 0.1 & 0.25 & 0.3 & 0.5 & 0.7 & 0.75 & & .0 & 1.25 & 4.88 \\
\hline \multicolumn{11}{|l|}{$\operatorname{Re}=40 ; v_{z}(0, z)$} \\
\hline 4 & 1.301 & 1.190 & 1.181 & 1.232 & 1.334 & 1.361 & & 487 & 1.593 & 1.981 \\
\hline 8 & 1.136 & 1.096 & 1.111 & 1.206 & 1.319 & 1.348 & & 480 & 1.589 & 1.981 \\
\hline 12 & 1.072 & 1.079 & 1.097 & 1.196 & 1.312 & 1.341 & & 476 & 1.587 & 1.980 \\
\hline 16 & 1.046 & 1.071 & 1.090 & 1.191 & 1.308 & 1.338 & & 474 & 1.585 & 1.980 \\
\hline 20 & 1.034 & 1.066 & 1.086 & 1.187 & 1.306 & 1.336 & & 472 & 1.584 & 1.980 \\
\hline 24 & 1.028 & 1.063 & 1.083 & 1.185 & 1.304 & 1.334 & & $\$ 71$ & 1.583 & 1.980 \\
\hline 28 & 1.024 & 1.061 & 1.081 & 1.184 & 1.303 & 1.333 & & $\$ 71$ & 1.583 & 1.980 \\
\hline 32 & 1.021 & 1.060 & 1.080 & 1.182 & 1.302 & 1.332 & & 770 & 1.582 & 1.980 \\
\hline 36 & 1.020 & 1.058 & 1.078 & 1.181 & 1.301 & 1.331 & & 469 & 1.582 & 1.980 \\
\hline 40 & 1.018 & 1.057 & 1.077 & 1.181 & 1.301 & 1.331 & & 469 & 1.582 & 1.980 \\
\hline 44 & 1.017 & 1.057 & 1.077 & 1.180 & 1.300 & 1.330 & & 469 & 1.582 & 1.980 \\
\hline \multirow[t]{2}{*}{ Reference [35] } & 1.008 & 1.0484 & 1.074 & 1.1738 & 1.310 & 1.3263 & & 4664 & 1.5799 & 1.980 \\
\hline & 0.5 & 1.0 & & 5.0 & 7.0 & \multicolumn{2}{|c|}{9.0} & 11.0 & 56 \\
\hline \multicolumn{11}{|l|}{$R e=500 ; v_{z}(0, z)$} \\
\hline 4 & 1.278 & 1.220 & \multicolumn{2}{|c|}{1.286} & 1.389 & 1.470 & \multicolumn{2}{|c|}{1.538} & 1.596 & 1.980 \\
\hline 8 & 1.099 & 1.126 & \multicolumn{2}{|c|}{1.289} & 1.389 & 1.468 & \multicolumn{2}{|c|}{1.536} & 1.595 & 1.980 \\
\hline 12 & 1.062 & 1.124 & \multicolumn{2}{|c|}{1.283} & 1.384 & 1.464 & \multicolumn{2}{|c|}{1.533} & 1.593 & 1.980 \\
\hline 16 & 1.058 & 1.119 & \multicolumn{2}{|c|}{1.279} & 1.380 & 1.461 & \multicolumn{2}{|c|}{1.530} & 1.591 & 1.980 \\
\hline 20 & 1.055 & 1.116 & \multicolumn{2}{|c|}{1.276} & 1.378 & 1.459 & \multicolumn{2}{|c|}{1.529} & 1.589 & 1.980 \\
\hline 22 & 1.053 & 1.114 & \multicolumn{2}{|c|}{1.275} & 1.377 & 1.459 & \multicolumn{2}{|c|}{1.528} & 1.589 & 1.980 \\
\hline Reference [35] & 1.0563 & 1.1337 & \multicolumn{2}{|c|}{1.2827} & 1.3836 & 1.4655 & \multicolumn{2}{|c|}{1.5352} & 1.5955 & 1.980 \\
\hline
\end{tabular}
different axial positions: (a) $\operatorname{Re}=0$, (b) $\operatorname{Re}=40$, (c) $\operatorname{Re}=500$.

Inlet conditions: $v_{z}=1$ and $v_{r}=0$ (parallel flow). 
Table II. Convergence behavior of the longitudinal velocity component along the centerline of the duct for different axial positions: [correction made here after initial online publication] (a) $R e=0$, (b) $R e=40$, (c) $R e=500$.

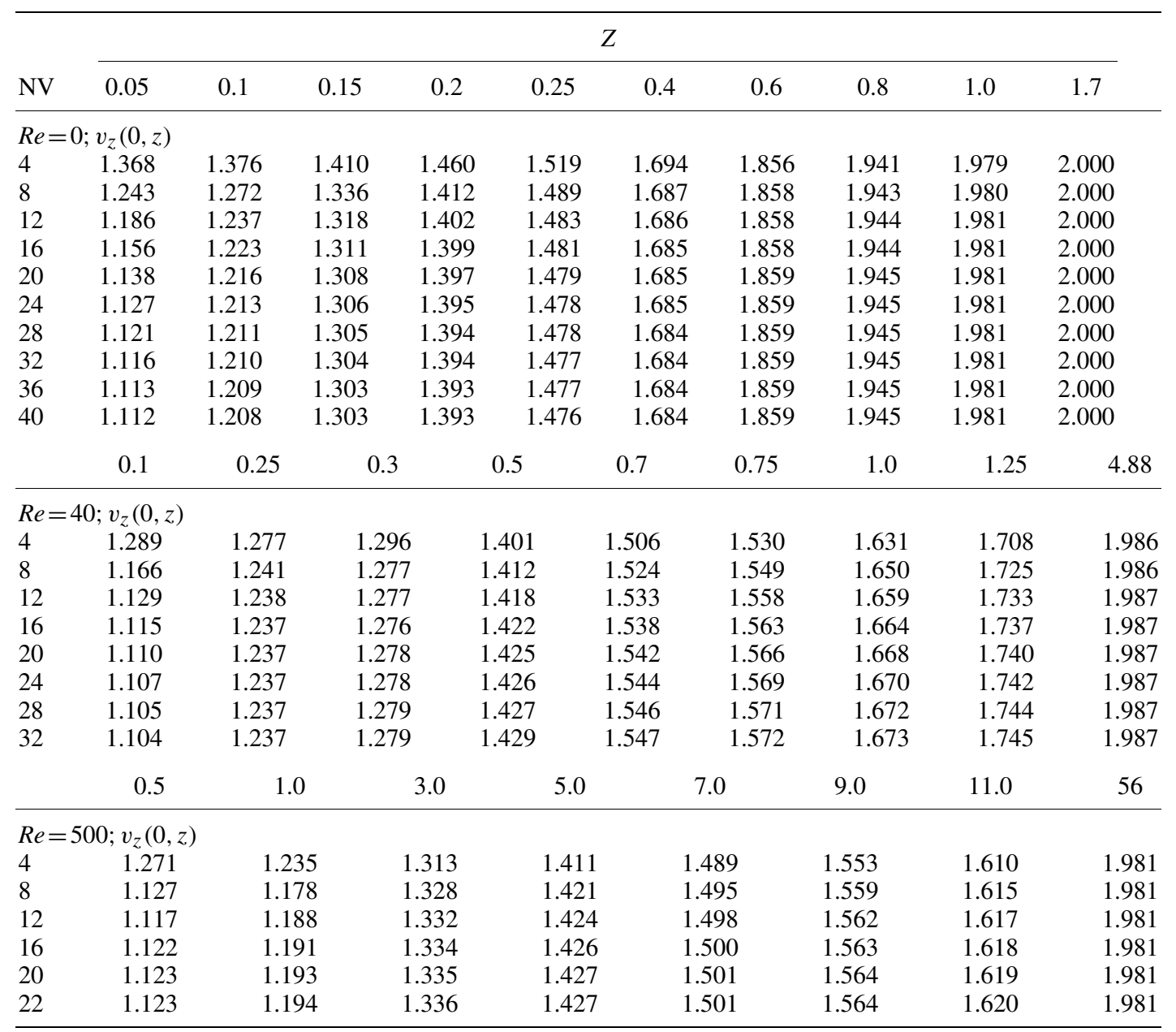

Inlet conditions: $v_{z}=1$ and $\omega=0$ (irrotational flow).

condition at the entrance: uniform parallel flow and irrotational flow. The computational code was developed in FORTRAN 90/95 programming language and implemented on a PENTIUM-IV $1.3 \mathrm{GHz}$ computer. The routine DBVPFD from the IMSL Library [36] was used to numerically handle the truncated version of the ordinary differential system, Equations (30)-(34), with a prescribed relative error target of $10^{-4}$ for the transformed potentials. For the velocity field, the results were produced with different truncation orders $(\mathrm{NV} \leqslant 60)$ so as to illustrate the convergence behavior, and for various values of Reynolds number, $R e=0,10,20,40,100,150,200,300,400$ and 500.

A set of tables was first prepared to illustrate the convergence behavior of the proposed eigenfunction expansion, in terms of the longitudinal velocity component at the duct centerline, for both inlet boundary conditions and for the different values of the governing parameter, Reynolds number. Table I(a) shows the convergence behavior of the longitudinal velocity component at the centerline of the circular tube for the case $R e=0$, considering boundary condition of uniform parallel flow at the channel inlet. This limiting case of the Navier-Stokes equation is of interest in different applications related to 'creeping flow', and also allows for a direct comparison with the results of Reference [35]. It can be observed that within the range of truncation order $\mathrm{NV}<48$ there is convergence of the GITT solution already to three significant digits. In addition, this table 


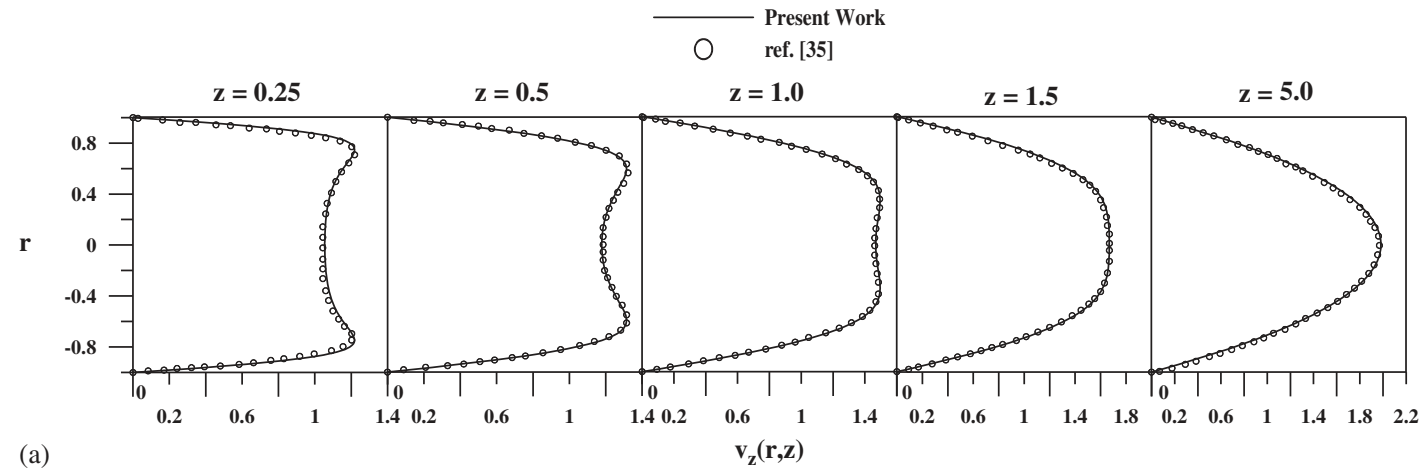

(a)
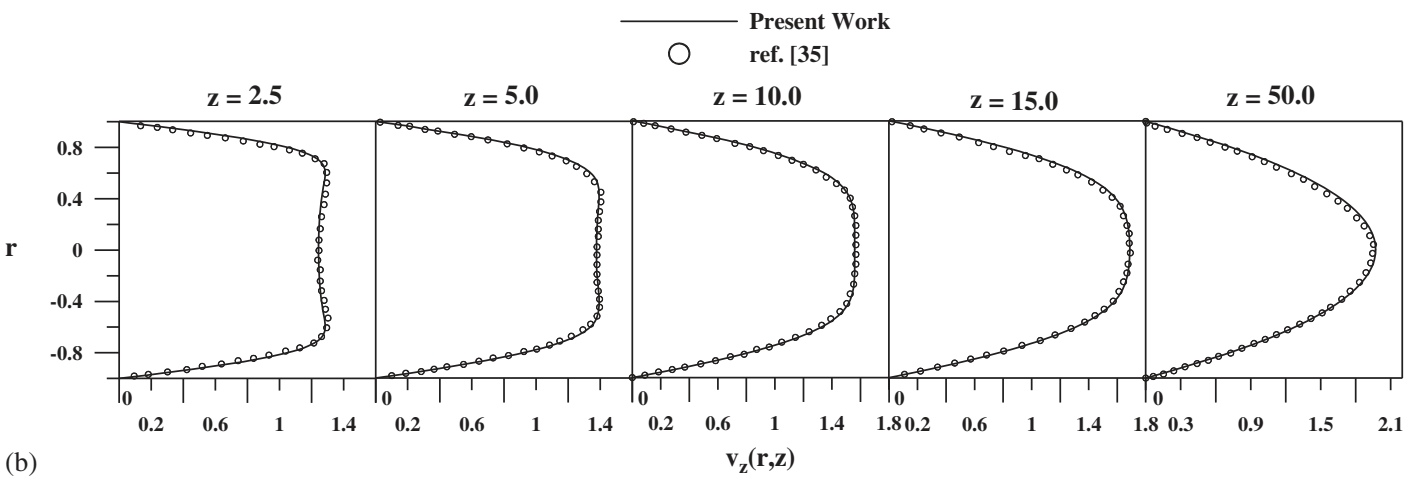

(b)

Figure 2. Velocity profile development $v_{z}(r, z)$ in circular tube, compared against numerical results of [35]. Uniform inlet flow. (a) $R e=40$ and (b) $R e=500$.

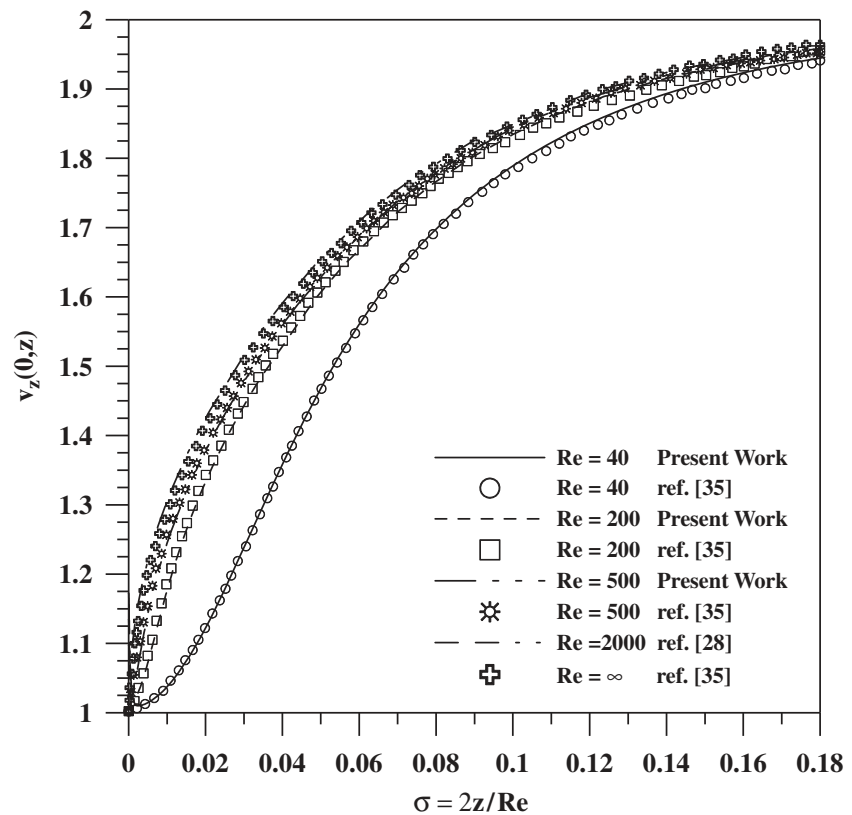

Figure 3. Development of longitudinal velocity component at the channel centerline, compared against numerical results of [35]. Uniform inlet flow. 
brings a comparison of the present results with those in Reference [35], and a reasonable agreement to roughly the third digit is achieved. A very similar convergence pattern was observed for the case $R e=40$, illustrated in Table $\mathrm{I}(\mathrm{b})$, together with the third digit agreement against the results in Reference [35], again for the parallel inlet flow situation. Table I(c) is aimed at demonstrating the effect of Reynolds number on the convergence behavior of the eigenfunction expansion, for $R e=500$, again with excellent behavior offering practically a four digits convergence for truncation orders as low as around $\mathrm{NV}=22$, and reconfirming the agreement to three significant digits with respect to previously published numerical results [35].

Tables II $(\mathrm{a}-\mathrm{c})$ present a similar set of results showing the convergence of the longitudinal velocity component in the centerline of the duct, for $R e=0,40$ and 500, respectively, but now considering the inlet condition of irrotational flow. The convergence of the solution is again fully reached to the third digit with $\mathrm{NV}<40$ in the expansion, but in this case of irrotational inlet flow, literature results are not readily available for direct comparison.

Figure 2(a) shows the development of the velocity profile $v_{z}(r, z)$ in the duct inlet region for $R e=40$ considering uniform velocity profile at the entrance, compared against the results obtained in [35]. An overall excellent agreement among the results is observed and the velocity profile exhibits the well-known 'overshooting' behavior close to the inlet. As discussed in [32], the velocity overshoots arise because a negative pressure gradient $\partial p / \partial z$ establishes near the wall region a very short distance downstream of the inlet $(z=0)$ section, whereas a positive pressure zone exists in the region near the centerline. Therefore, the flow near the centerline is not immediately accelerated and the velocity overshoots are formed. Closer to the inlet region, more evident is the overshoot, as can be seen from Figure 2(a). Also, Figure 2(b) shows the development of the velocity profile $v_{z}(r, z)$ for the case $R e=500$, again for parallel inlet flow. The same conclusions may be reached, but it should be noted that this case requires some changes on the parameter of scale compression to accelerate the convergence of the boundary value problem for the transformed potentials, according to the routine DBVPFD of the IMSL system. Again an excellent agreement among the results is observed and the velocity overshoots are less pronounced.

In Figure 3 we display graphs of the longitudinal velocity component $v_{z}(0, z)$ along the centerline of the duct as a function of the dimensionless coordinate $\sigma=(2 z / R e)$, together with comparisons against the results reported in [35], for $R e=40,200,500$ and infinity (representative of the boundary layer formulation). The attainment of the fully developed flow region is observed, besides the tendency toward the boundary-layer results for increasing Reynolds number. The results found through the GITT are in excellent graphical agreement with the results of [35].

Figure 4 again exhibits the longitudinal velocity component along the channel centerline, but this time for both inlet flow conditions and $R e=40$ and 500 . Here, the objective is to compare the effects of the two inlet flow boundary conditions in relation to the boundary-layer behavior, as also recently obtained via integral transforms [28]. For direct comparison against the original boundary-layer solution [28], the dimensionless axial coordinate is taken as $X^{+}=(z / 2 R e)$. As for the parallel plate channel situation [13], the solution of the full Navier-Stokes equations considering null vorticity at the entrance is closer to the solution obtained through the boundary layer formulation in the vicinity of the duct inlet, especially for higher Reynolds numbers.

Figure 5 provides a comparison of the transversal velocity component profiles reported in the present work with the results obtained from the implementation of the system of equations given in Reference [28] using the boundary-layer model for a circular duct, also applying GITT. The differences between the solutions from the boundary-layer model and from the full Navier-Stokes equations are also quite evident for the radial velocity component, with a less disturbed flow pattern at the channel inlet region being predicted by the boundary-layer approximation, which neglects the radial pressure gradients. As expected, the two solutions approach each other for increasing axial position toward the fully developed region.

Figures $6(a, b)$ offer an analysis of the transversal velocity component profiles comparing the two inlet flow conditions considered here, for Reynolds numbers equal to 40 and 500, respectively. For both Reynolds numbers, the imposition of irrotational flow at the channel entrance leads to a shorter distance for the flow development, with a more marked difference for the lower Reynolds numbers. 


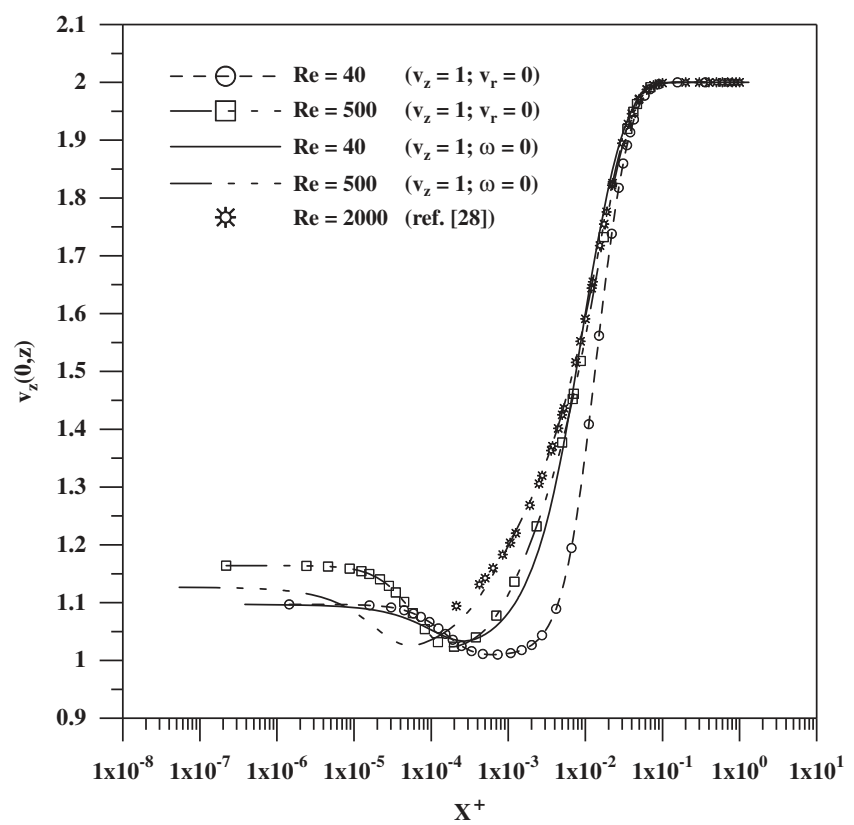

Figure 4. Comparison of longitudinal velocity component at the channel centerline for two types of inlet boundary condition: uniform velocity profile $\left(v_{z}=1 ; v_{r}=0\right)$ and irrotational flow $\left(v_{z}=1 ; \omega=0\right)$.

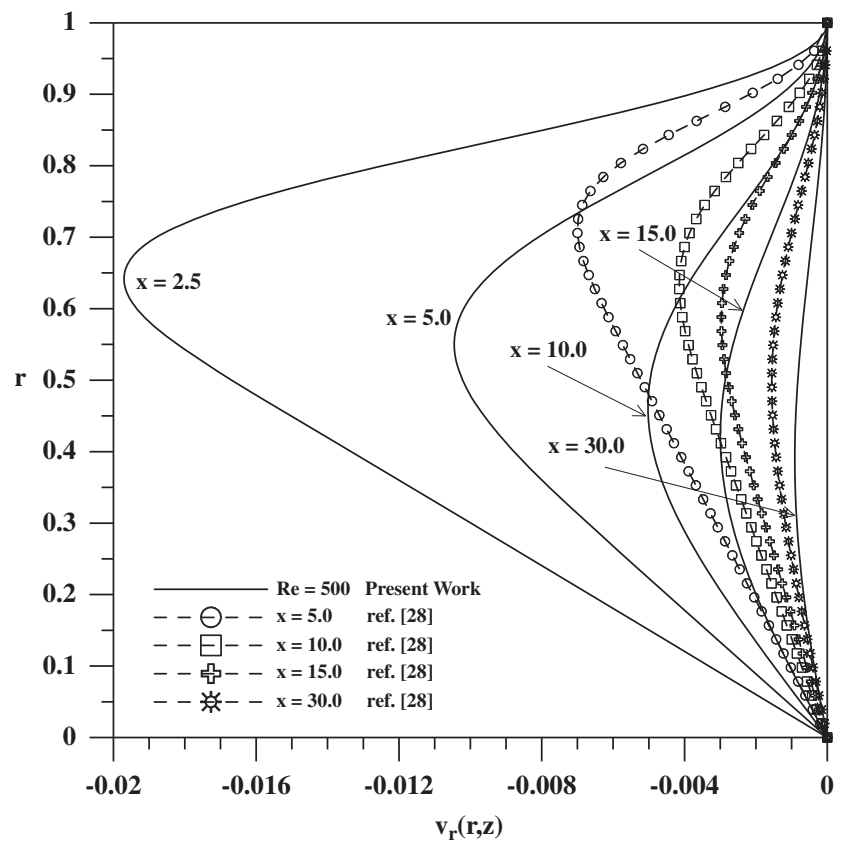

Figure 5. Comparison of Navier-Stokes $(R e=500)$ and boundary-layer [28] solutions for the radial velocity component profiles. Uniform velocity profile $\left(v_{z}=1 ; v_{r}=0\right)$.

Another comparison of the developing flow patterns for the two inlet conditions is provided in Figures $7(\mathrm{a}, \mathrm{b})$, which show the developing velocity profile $v_{z}(r, z)$ along the axial position of the duct for Reynolds number equal to 40 and 500, respectively. Such as observed in the solution of the Navier-Stokes equations for a parallel plates duct [13], when the inlet condition of the duct is of irrotational flow, the velocity overshoots are hardly observable. For Reynolds number equal 

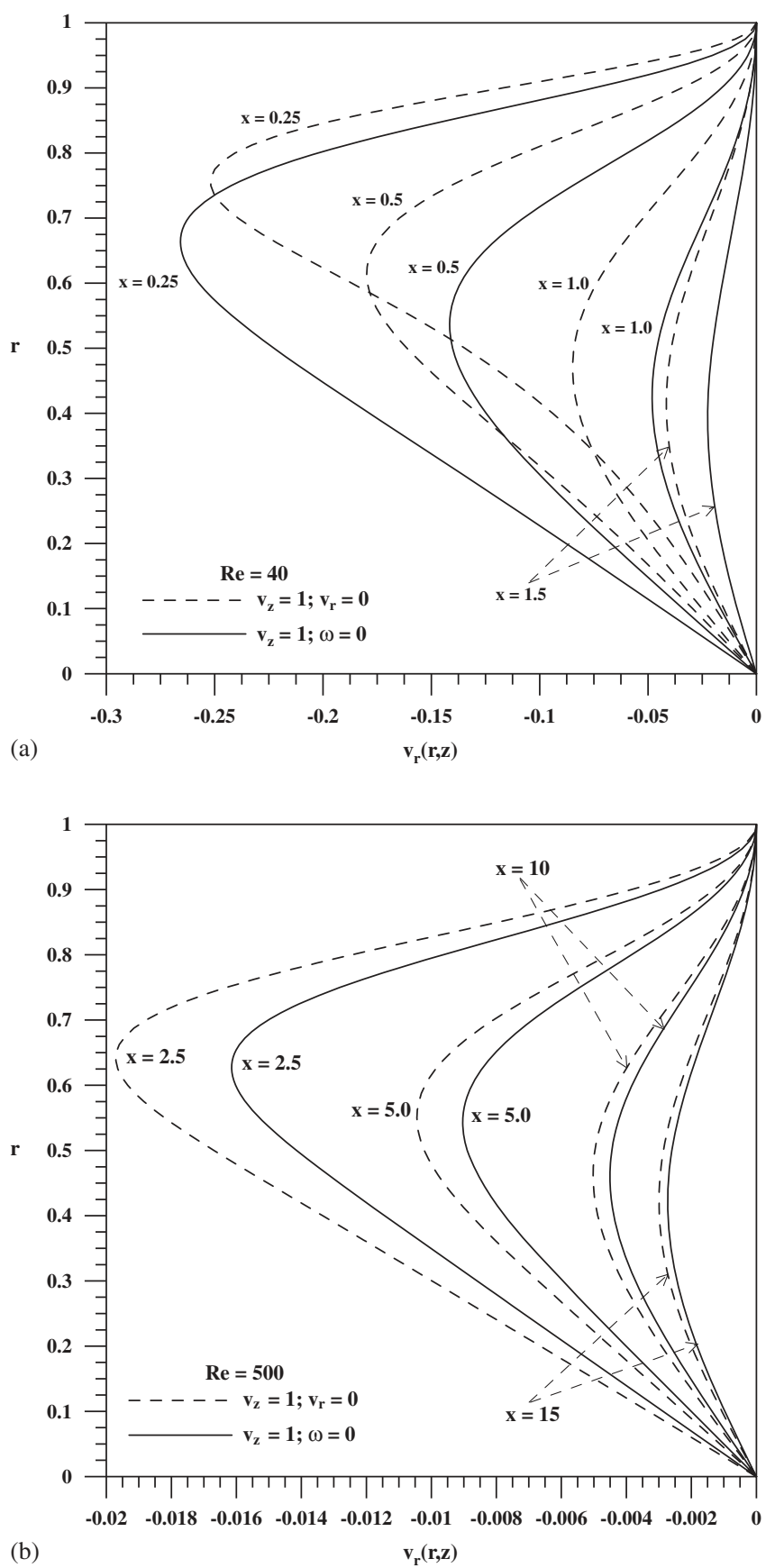

Figure 6. Radial velocity component profiles for two types of inlet boundary condition: uniform velocity profile $\left(v_{z}=1 ; v_{r}=0\right)$ and irrotational flow $\left(v_{z}=1 ; \omega=0\right)$. (a) $R e=40$ and (b) $R e=500$.

to 40 , very close to the duct entrance, this behavior is still noticeable to the graph scale. Once more, from these figures, it is clearly observable that the fully developed flow is reached within a shorter channel length when the Navier-Stokes equations are complemented by the limiting inlet condition of irrotational flow.

Finally, Figures $8(a, b)$ illustrates the results for the product of the Fanning friction factor and Reynolds number as a function of the dimensionless axial position, $X^{+}$. It can be observed, as expected, that the product $\mathrm{f} \cdot R e$ decreases until the fully developed region is reached, where this 


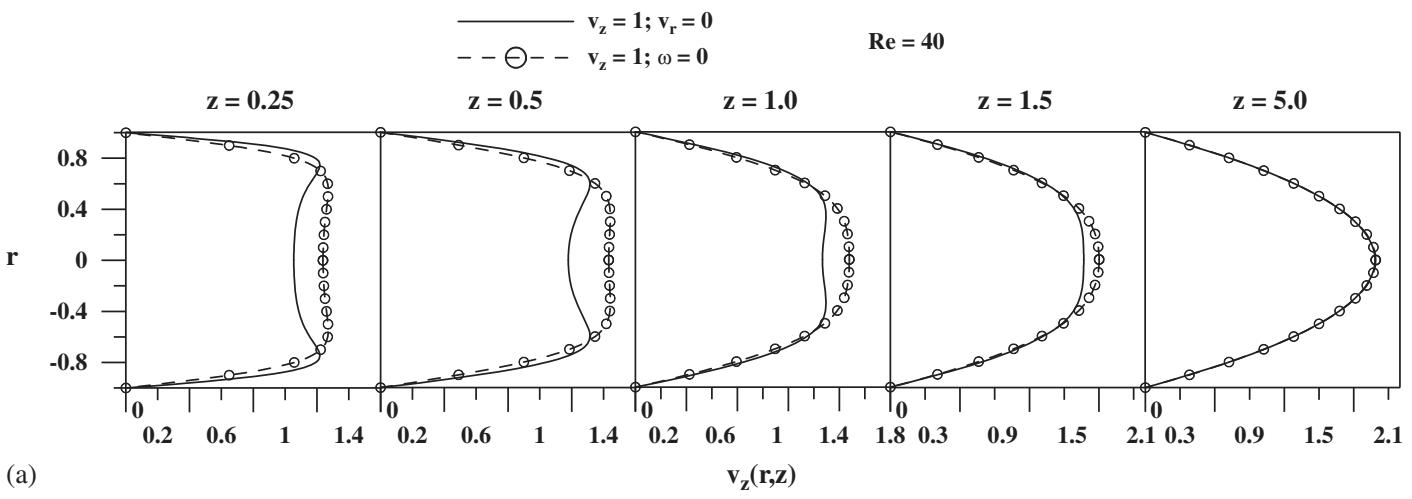

(a)

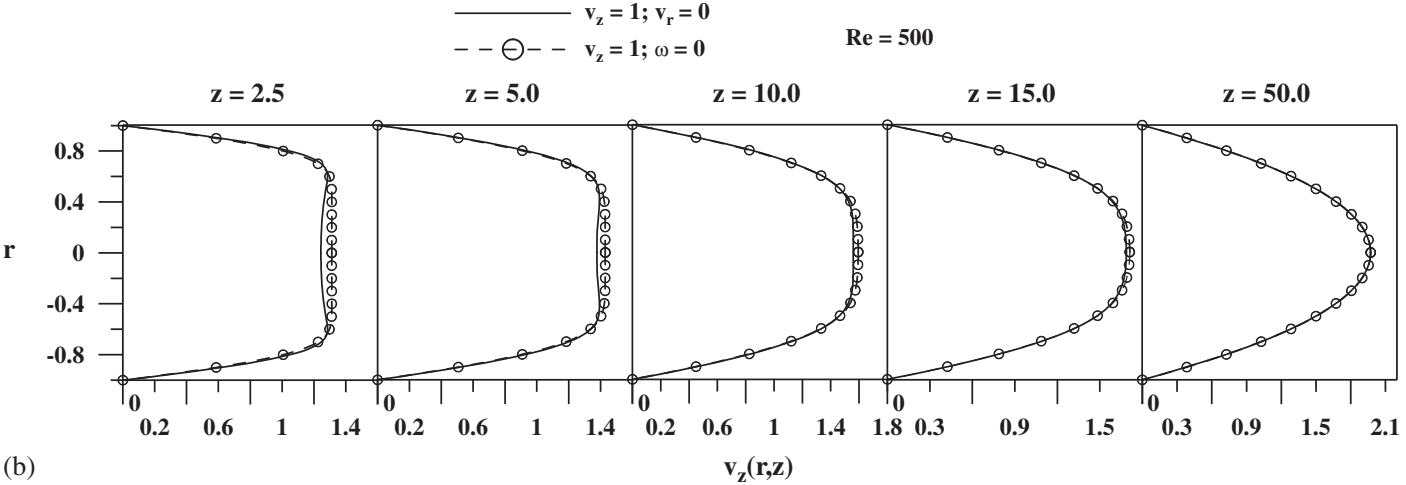

Figure 7. Comparison of velocity profile development $v_{z}(r, z)$ in the inlet region of the duct for two types of inlet boundary condition: uniform velocity profile $\left(v_{z}=1 ; v_{r}=0\right)$ and irrotational flow $\left(v_{z}=1 ; \omega=0\right)$. (a) $\operatorname{Re}=40$ and (b) $\operatorname{Re}=500$.

parameter assumes its well-known asymptotic value. Also, it should be recalled that the product f. Re presents higher values in the entrance region of the circular tube due to the obviously higher velocity gradients experienced by the fluid in this region, and despite the slower convergence of the eigenfunction expansions very close to the inlet, the results represented were revealed to be fully converged to the graph scale, offering a set of reference results for this quantity of practical interest.

\section{CONCLUSIONS}

The integral transform method was applied in the hybrid numerical-analytical solution of the Navier-Stokes equations in cylindrical coordinates. The streamfunction formulation was preferred and the solid cylinder geometry was pursued for the purpose of handling the singularity at the channel centerline. Adoption of a recently introduced eigenvalue problem allowed for a straightforward application of the GITT approach in the streamfunction formulation for such a full cylindrical region. Hydrodynamically developing flow in a circular tube was chosen as a test case for the present methodology extension. Numerical results for the velocity components and for the product of the Fanning friction factor-Reynolds number were then produced for two different inlet conditions, uniform and irrotational flow. The excellent agreement of the present results with previously reported numerical implementations demonstrates the consistency of the proposed methodology and constructed code. The same eigenfunction expansion employed here for laminar flow may be equally utilized to produce results for the turbulent flow situation and/or non-Newtonian fluids, by considering classical algebraic turbulence models and/or constitutive equations in the problem 


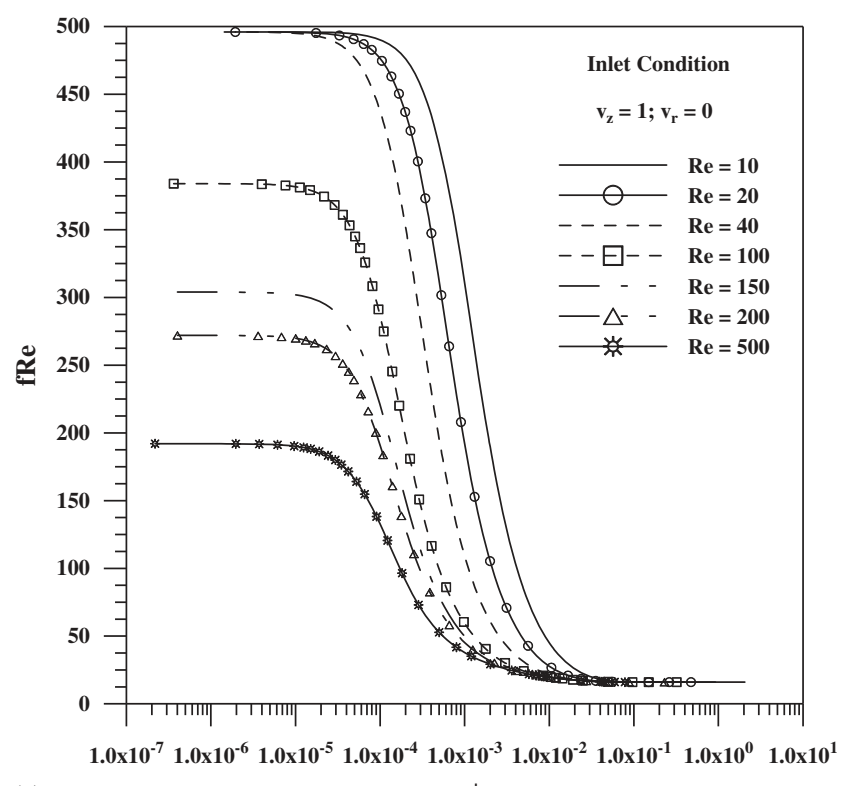

(a)

$\mathbf{X}^{+}$

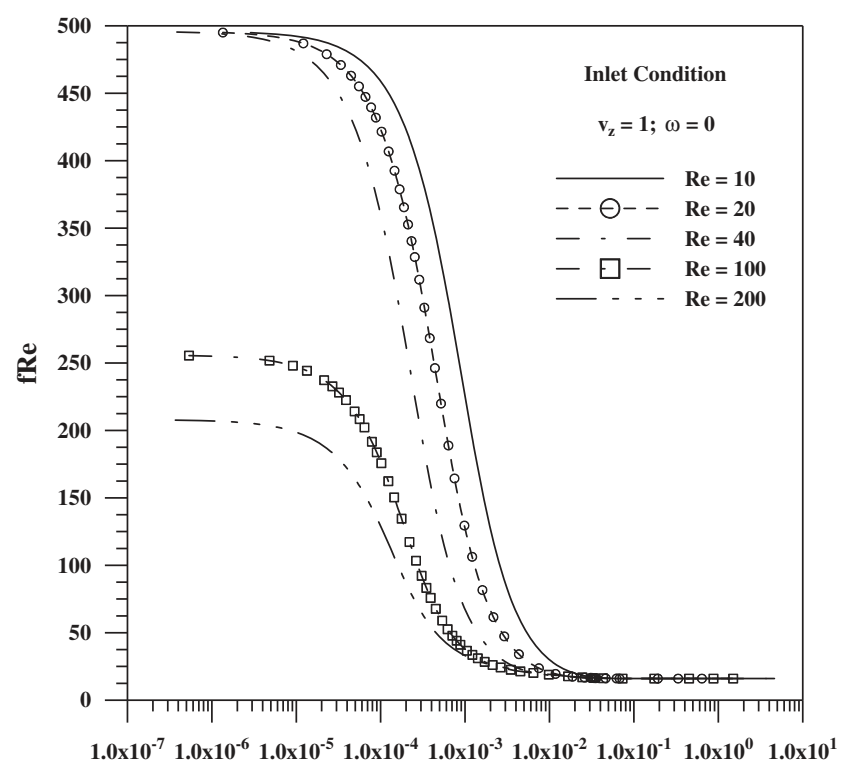

(b)

$$
\mathbf{X}^{+}
$$

Figure 8. Development of the product $\mathrm{f} R e$ along the dimensionless axial coordinate $X^{+}$for various Reynolds number. Inlet condition: (a) $v_{z}=1 ; v_{r}=0$ and (b) $v_{z}=1 ; \omega=0$.

formulation, such as previously implemented for parallel plates channels $[6,16]$, but in principle maintaining the same auxiliary problem structure.

\section{NOMENCLATURE}

$A_{i j}, B_{i j k}, C_{i j k} \quad$ coefficients defined by Equations (35), (36) and (37), respectively

$c$

$D_{i j k}, E_{i j}$

$F_{i j}$ scale contraction parameter

coefficients defined by Equations (38) and (39), respectively

coefficient defined by Equation (40) 
$f \quad$ Fanning friction factor

$\bar{g}_{i} \quad$ coefficient defined by Equation (41)

$J_{1} \quad$ Bessel function of the first kind and order one

$N_{i} \quad$ normalization integral

$\mathrm{NV}$ truncation order

$p^{*}, p \quad$ pressure field, dimensional and dimensionless, respectively

$r^{*}, r \quad$ radial coordinate, dimensional and dimensionless, respectively

Re Reynolds number

$r_{w} \quad$ tube radius

$u_{0} \quad$ inlet and average velocity

$v_{r}^{*}, v_{r} \quad$ radial velocity component, dimensional and dimensionless, respectively

$v_{z}^{*}, v_{z} \quad$ longitudinal velocity component, dimensional and dimensionless, respectively

$X^{+} \quad$ dimensionless longitudinal coordinate

$X_{i}(r) \quad$ eigenfunctions

$z^{*}, z \quad$ longitudinal coordinate, dimensional and dimensionless, respectively

$W \quad$ solution vector used for the computational procedure

\section{Greek letters}

$\begin{array}{ll}\eta & \text { coordinate in domain transformation } \\ \lambda_{i} & \text { eigenvalues } \\ v & \text { kinematic viscosity } \\ \rho & \text { fluid density } \\ \sigma & \text { dimensionless longitudinal coordinate } \\ \tau_{\mathrm{w}} & \text { wall shear stress } \\ \phi & \text { filtered streamfunction } \\ \bar{\phi}_{i}(z) & \text { transformed potential } \\ \psi & \text { streamfunction } \\ \psi_{\infty} & \text { fully developed streamfunction } \\ \omega & \text { vorticity }\end{array}$

\section{Subscripts and Superscripts}

$\begin{array}{ll}i, j, k, m & \text { order from eigenvalue problems } \\ \mathrm{w} & \text { referred to the wall } \\ - & \text { integral transformed quantities } \\ \infty & \text { referred to fully developed situation }\end{array}$

\section{ACKNOWLEDGEMENTS}

The authors would like to acknowledge the financial support provided by CNPq and CENPES-Petrobras.

\section{REFERENCES}

1. Minkowycz WJ, Sparrow EM, Murthy JY. Handbook of Numerical Heat Transfer (2nd edn). Wiley: New York, U.S.A., 2006.

2. Cotta RM. Hybrid numerical-analytical approach to nonlinear diffusion problems. Numerical Heat Transfer, Part B-Fundamentals 1990; 17:217-226.

3. Cotta RM. Integral Transforms in Computational Heat and Fluid Flow. CRC Press: Boca Raton, FL, U.S.A., 1993.

4. Cotta RM. Benchmark results in computational heat and fluid flow: the integral transform method. International Journal of Heat and Mass Transfer (Invited Paper) 1994; 37(Suppl. 1):381-393.

5. Cotta RM. The Integral Transform Method in Thermal and Fluid Sciences and Engineering. Begell House: New York, U.S.A., 1998. 
6. Santos CAC, Quaresma JNN, Lima JA. Benchmark Results for Convective Heat Transfer in Ducts: The Integral Transform Approach. ABCM Mechanical Sciences Series. Editora E-Papers: Rio de Janeiro, Brazil, 2001.

7. Cotta RM, Santos CAC, Quaresma JNN, Perez-Guerrero JS. Hybrid integral transforms in convection-diffusion: recent applications in internal flow simulation, invited lecture. Proceedings of the 4th International Conference on Computational Heat and Mass Transfer, 4th ICCHMT, vol. 1, Paris-Cachan, France, 2005; 153-164.

8. Cotta RM, Mikhailov MD. Hybrid methods and symbolic computations. In Handbook of Numerical Heat Transfer (2nd edn), Chapter 16, Minkowycz WJ, Sparrow EM, Murthy JY (eds). Wiley: New York, U.S.A., 2006; 493-522.

9. Campos Silva JB, Cotta RM, Aparecido JB. Analytical solutions to simultaneously developing laminar flow inside parallel-plates channel. International Journal of Heat and Mass Transfer 1992; 35:887-895.

10. Perez-Guerrero JS, Cotta RM. Integral transform method for Navier-Stokes equations in stream function-only formulation. International Journal for Numerical Methods in Fluids 1992; 15:399-409.

11. Carvalho TMB, Cotta RM, Mikhailov MD. Flow development in entrance region of ducts. Communications in Numerical Methods in Engineering 1993; 9:503-509.

12. Machado HA, Cotta RM. Integral transform method for boundary layer equations in simultaneous heat and fluid flow problems. International Journal of Numerical Methods for Heat and Fluid Flow 1995; 5:225-237.

13. Perez-Guerrero JS, Cotta RM. Integral transform solution of developing laminar duct flow in Navier-Stokes formulation. International Journal for Numerical Methods in Fluids 1995; 20:1203-1213.

14. Figueira da Silva E, Cotta RM. Benchmark results for internal forced convection through integral transformation. International Communications in Heat and Mass Transfer 1996; 23:1019-1029.

15. Perez-Guerrero JS, Cotta RM. Benchmark integral transform results for flow over a backward-facing step. Computers and Fluids 1996; 25:527-540.

16. Lima JA, Perez-Guerrero JS, Cotta RM. Hybrid solution of the averaged Navier-Stokes equations for turbulent flow. Computational Mechanics 1997; 19:297-307.

17. Quaresma JNN, Cotta RM. Integral transform method for the Navier-Stokes equations in steady three-dimensional flow. Proceedings of the 10th ISTP-International Symposium on Transport Phenomena, Kyoto, Japan, 1997; 281-287.

18. Cotta RM, Pimentel LCG. Developing turbulent duct flow: hybrid solution via integral transforms and algebraic models. International Journal of Numerical Methods for Heat and Fluid Flow 1998; 8:10-26.

19. Figueira da Silva E, Cotta RM. Mixed convection within vertical parallel-plates: hybrid solution by integral transforms. Numerical Heat Transfer, Part A-Applications 1998; 33:85-106.

20. Pereira LM, Perez-Guerrero JS, Cotta RM. Integral transformation of the Navier-Stokes equations in cylindrical Geometry. Computational Mechanics 1998; 21:60-70.

21. Figueira da Silva E, Perez-Guerrero JS, Cotta RM. Integral transform solution of boundary layer equations in streamfunction-only formulation. International Journal of Non-Linear Mechanics 1999; 34:51-61.

22. Machado HA, Cotta RM. Analysis of internal convection with variable physical properties via integral transformation. Numerical Heat Transfer, Part A-Applications 1999; 36:699-724.

23. Perez-Guerrero JS, Quaresma JNN, Cotta RM. Simulation of laminar flow inside ducts of irregular geometry using integral transforms. Computational Mechanics 2000; 25:413-420.

24. Leal MA, Machado HA, Cotta RM. Integral transform solutions of transient natural convection in enclosures with variable fluid properties. International Journal of Heat and Mass Transfer 2000; 43:3977-3990.

25. Pereira LM, Perez-Guerrero JS, Cotta RM. Analysis of laminar forced convection in annular ducts using integral transforms. Hybrid Methods in Engineering 2000; 2:221-232.

26. Ramos R, Perez-Guerrero JS, Cotta RM. Stratified flow over a backward facing step: hybrid solution by integral transforms. International Journal for Numerical Methods in Fluids 2001; 35:173-197.

27. de Lima GGC, Santos CAC, Haag A, Cotta RM. Integral transform solution of internal flow problems based on Navier-Stokes equations and primitive variables formulation. International Journal for Numerical Methods in Engineering 2007; 69:544-561.

28. Paz SPA, Macêdo EN, Quaresma JNN, Cotta RM. Eigenfunction expansion solution for boundary-layer equations in cylindrical coordinates: simultaneously developing flow in circular tubes. Numerical Heat Transfer, Part AApplications 2007; 52:1123-1149.

29. Fedele F, Hitt DL, Prabhu RD. Revisiting the stability of pulsatile pipe flow. European Journal of Mechanics B/Fluids 2005; 24:237-254.

30. Shah RK, London AL. Laminar flow forced convection in ducts. Advances in Heat Transfer, Supplement 1. Academic Press: New York, U.S.A., 1978.

31. Kakaç S. Handbook of Single-phase Convective Heat Transfer. Wiley: New York, U.S.A., 1987.

32. Sparrow EM, Lin SH, Lundgren TS. Flow development in the hydrodynamic entrance region of tubes and ducts. Physics of Fluids 1964; 7:338-347.

33. Hornbeck RW. Laminar flow in the entrance region of a pipe. Applied Scientific Research, Section A 1964; 13:224-232.

34. Liu J. Flow of a Bingham fluid in the entrance region of an annular tube. M.S. Thesis, University of WisconsinMilwaukee, U.S.A., 1974.

35. Friedmann M, Gillis J, Liron N. Laminar flow in a pipe at low and moderate Reynolds numbers. Applied Scientific Research 1968; 19:426-438.

36. IMSL Library. MATH/LIB, Houston, TX, U.S.A., 1991. 